\title{
Session 12: Closing Plenary Session
}

Friday 16th April 2010. Moderator: Mark C. Glassy

[16.50-17.10]

'HAH 2010 conference overview and summary'

Don Capra

Oklahoma Medical Research Foundation, USA

Abstract not provided.

[17.10]

Closing remarks by: Mark C. Glassy

HAH 2010 Conference Chairman and Nascent Biolog-

ics Inc., San Diego, California, USA 